\title{
A VISION ON THE FUTURE OF ARTICULAR CARTILAGE REPAIR
}

\author{
M. Cucchiarini ${ }^{1,7}$, H. Madry ${ }^{1,7}$, F. Guilak ${ }^{2,7}$, D.B. Saris ${ }^{3}$, M.J. Stoddart4,7, M. Koon Wong ${ }^{5}$ and P. Roughley ${ }^{6, *}$ \\ ${ }^{1}$ Center of Experimental Orthopaedics, Saarland University, Homburg, Germany \\ ${ }^{3}$ Department of Biomedical Engineering, Duke University Medical Centre, Durham, NC, USA \\ ${ }^{3}$ Department of Orthopaedics, University Medical Center, Utrecht, The Netherlands \\ ${ }^{4}$ AO Research Institute Davos, Davos Platz, Switzerland \\ ${ }^{5}$ Department of Orthopaedics, Singapore General Hospital, Singapore \\ ${ }^{6}$ Genetics Unit, Shriners Hospital for Children, Montreal, Canada \\ ${ }^{7}$ Collaborative Research Partner, Acute Cartilage Injury Program of the AO Foundation, Davos, Switzerland
}

\begin{abstract}
An AO Foundation (Davos, Switzerland) sponsored workshop "Cell Therapy in Cartilage Repair" from the Symposium "Where Science meets Clinics" (September 5-7, 2013, Davos) gathered leaders from medicine, science, industry, and regulatory organisations to debate the vision of cell therapy in articular cartilage repair and the measures that could be taken to narrow the gap between vision and current practice. Cell-based therapy is already in clinical use to enhance the repair of cartilage lesions, with procedures such as microfracture and articular chondrocyte implantation. However, even though long term follow up is good from a clinical perspective and some of the most rigorous randomised controlled trials in the regenerative medicine/orthopaedics field show beneficial effect, none of these options have proved successful in restoring the original articular cartilage structure and functionality in patients so far. With the remarkable recent advances in experimental research in cell biology (new sources for chondrocytes, stem cells), molecular biology (growth factors, genes), biomaterials, biomechanics, and translational science, a combined effort between scientists and clinicians with broad expertise may allow development of an improved cell therapy for cartilage repair. This position paper describes the current state of the art in the field to help define a procedure adapted to the clinical situation for upcoming translation in the patient.
\end{abstract}

Keywords: Articular cartilage repair, microfracture, articular chondrocyte implantation, tissue engineering, neomaterials, cell and gene therapy, articular chondrocytes, progenitor cells.

\footnotetext{
*Address for correspondence:

P. J. Roughley

Genetics Unit, 1529 Cedar Avenue

Shriners Hospital for Children

Montreal, Quebec, H3G 1A6, Canada

Telephone Number: 1-514-282-7156

FAX Number: 1-514-842-5581

E-mail: proughley@shriners.mcgill.ca
}

\section{Introduction}

Articular cartilage defects are common clinical problems that remain challenging as none of the current reconstructive surgical options are capable of restoring the original biological and mechanical properties of normal articular cartilage, particularly with larger defects.

Cartilage repair techniques are most commonly used with acute focal defects rather than the widespread cartilage loss of late stage osteoarthritis. Such focal lesions are often associated with excessive joint loading, which may be either be acute (e.g., due to high impact trauma) or cumulative (e.g., due to overuse associated with sport) in nature. Regardless of the origin, the consequence is the production of proteolytic enzymes by the chondrocytes at the site of injury that destroy the cartilage extracellular matrix and so propagate the defect.

Articular cartilage defects may be classified as either chondral or osteochondral (Fig. 1), although only $5 \%$ are osteochondral (Hjelle et al., 2002). Articular cartilage repair is primarily designed to treat chondral lesions (the type of lesions that do not extend deep into the subchondral bone). Such chondral lesions can either be classified as partial or full-thickness. As partial thickness lesions are always debrided down to the subchondral bone by removing the remaining articular cartilage including the calcified layer, they become in principle full-thickness lesions, and these are therefore the focus of clinical cartilage repair. However, the ideas presented here are in theory also applicable for osteochondral lesions, with the modification that the problem of the subchondral bone has to be also addressed (Orth et al., 2013).

\section{Current Status, Advantages, and Limitations}

Various cell-based therapies are available in the clinics to enhance the repair of cartilage defect (Bekkers et al., 2009). The major treatment options nowadays include marrow stimulation techniques, such as microfracture, indicated for small lesions $\left(<2.5 \mathrm{~cm}^{2}\right.$ defect area), and articular chondrocyte implantation (ACI) (Brittberg, 2008) employed for large lesions $\left(>2.5 \mathrm{~cm}^{2}\right.$ defect area) (Fig. $2)$. In theory, microfracture is attractive, as it is a onestep, simple, low-cost approach that stimulates a natural wound healing response involving bioactive factors and endogenous stem cells from the marrow space (Steadman et al., 2001). ACI has the advantage of using cells that form adult cartilage, i.e., the articular chondrocytes, and thus do not require cues to induce differentiation. 


\section{Chondral defects}

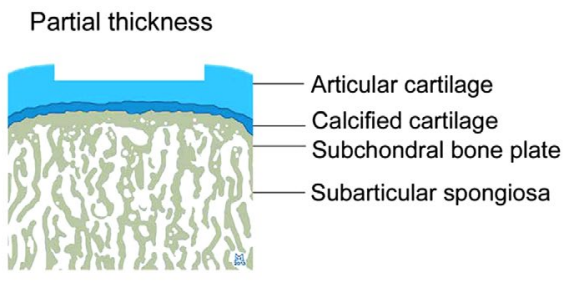

Full thickness

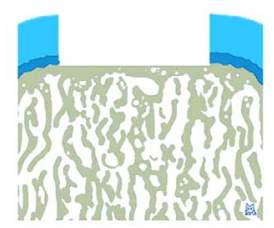

\section{Osteochondral defect}

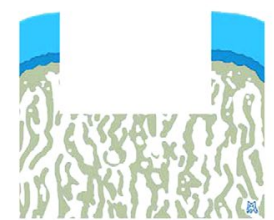

Fig. 1. Classification of cartilage defects. Chondral defects affect only the articular cartilage layer, in contrast to osteochondral defects that reach into the subchondral bone. A partial thickness lesion does not reach down to the subchondral bone, while in full thickness chondral defects the subchondral bone plate is exposed but not disturbed.

Furthermore, under current advanced-therapy-medicinalproduct (ATMP) regulations in the European Union, they have a known safety profile and efficacy parameters (Brittberg, 2010).

The current clinical challenge is that hyaline articular cartilage regeneration (both at the structural and functional/ mechanical levels) has not been achieved in adults yet using either technique, with the formation of a fibrocartilaginous repair tissue instead of the original hyaline cartilage being a common outcome (Madry et al., 2011). It is also noteworthy that no universal technique is available regardless of the type (chondral versus osteochondral) and size of the defects. Additional obstacles are further encountered with the use of these procedures. First, in the microfracture approach, there is no possibility to directly control the chondrogenic process, which may depend on a number of local as well as systemic factors that can be patient-specific. On the other hand, ACI is a two-step, expensive and complex protocol that requires expansion of chondrocytes in vitro, a process where the cells tend to dedifferentiate and lose their phenotype (after several population doublings). In addition, different membranes and cell seeding techniques are currently in use for ACI, without consensus on how to seed the cells (and how many cells are needed), on the timing of seeding for implantation, or on the optimal membrane for cell containment (most

\section{Marrow stimulation}

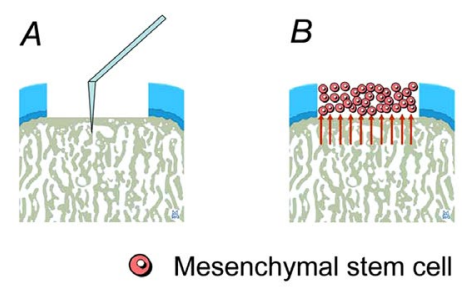

\section{Autologous chondrocyte implantation}

C

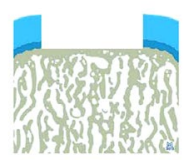

$D$

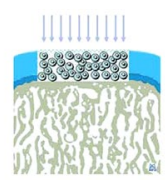

๑) Articular chondrocyte
Fig. 2. Overview of marrow stimulation and autologous chondrocyte implantation. Marrow stimulation techniques include microfracture $(\mathbf{A}, \mathbf{B})$, subchondral drilling, and abrasion arthroplasty. These techniques are indicated for symptomatic small articular cartilage defects. The overriding principle of these measures is to establish a communication of the cartilage defect with the subchondral bone marrow, either by focal perforation of the cement line with awls (microfracture) (A) or drill bits (subchondral drilling) or by a generalised and limited abrasion of the subchondral bone plate with burrs (abrasion arthroplasty). Prior to these procedures, the borders of the defect are debrided to reach stable and vertically oriented peripheral margins, and the entire calcified cartilage layer is removed from the base of the defect. Once a connection between the subchondral bone marrow and the cartilage defect has been achieved, pluripotent mesenchymal stem cells from the subchondral bone marrow migrate into the defect (B), differentiate into chondrocytes, and form a cartilaginous repair tissue. Autologous articular chondrocyte implantation (ACI) $(\mathbf{C}, \mathbf{D})$ is indicated for symptomatic large articular cartilage defects. The guiding principle of ACI is to implant autologous articular chondrocytes seeded onto a biodegradable membrane into the defect. ACI is performed several weeks after an initial cartilage biopsy. The defect has been similarly prepared as for marrow stimulation techniques, including removal of the entire calcified cartilage layer $(\mathbf{C})$. The integrity of the subchondral bone, however, is not disturbed. Instead, the membrane carrying the articular chondrocytes is implanted (D) and carefully secured to stay within the defect, forming the basis for a cartilaginous repair tissue.

membranes are based on type-I/-III collagen but there is no clear definition on their specific requirements, such as their pore size, biodegradability, or the use of hydrogels versus solid scaffolds). Medicinal product regulations provide guidance on cell dose, administration method and scaffold components, which is an important step forward in 

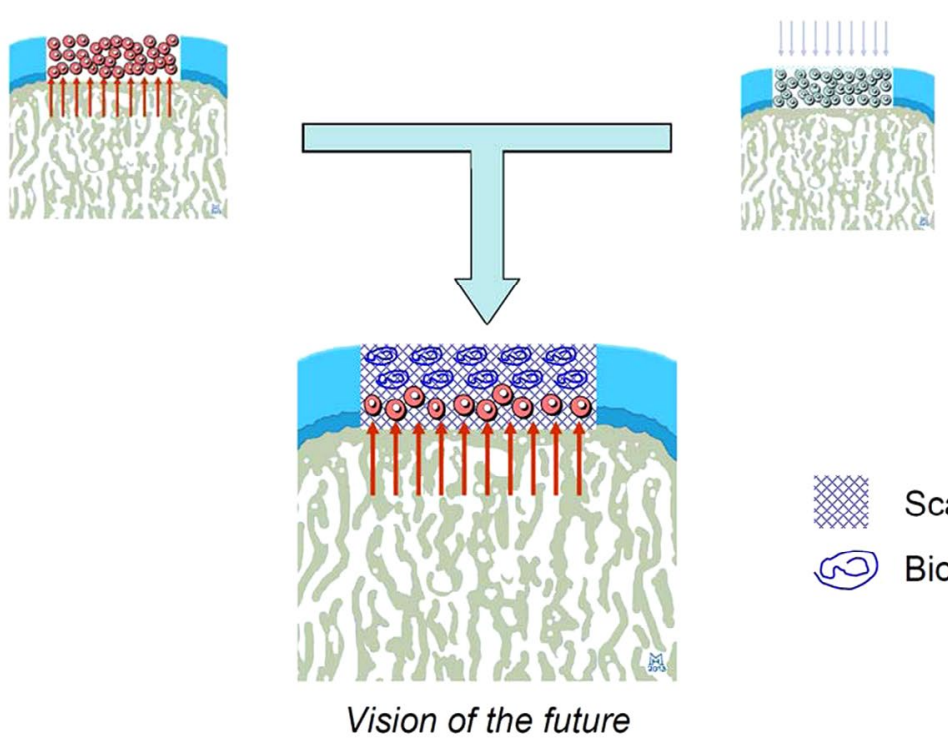

Scaffold

Biological signal

Fig. 3. A vision of the future of cartilage repair. A vision strategy combining components of the clinically successful approaches of marrow stimulation and autologous chondrocyte implantation (ACI) may include the use of a scaffold material supplemented with biological signals (e.g., growth factors or genes) to be implanted into a full-thickness cartilage defect. The defect is prepared by a marrow stimulation technique to grant access of the MSCs from the subchondral bone. These cells may populate the scaffold and, under the influence of the biological signals provided, undergo chondrogenesis to promote cartilage regeneration.

establishing clarity and selecting truly performing solutions from amongst the plentiful options previously available.

While all these factors need to be taken into consideration, these observations show the crucial need to establish a new, adapted option for articular cartilage repair that takes into account the clinical situation.

\section{Vision for the Future}

\section{Measures to Narrow the Gap between Vision and Current Practice}

\section{The vision}

The vision for cartilage repair is to bring forward and improve both existing procedures (microfracture, ACI) by bridging the gap between scientific knowledge and clinical experience in order to develop a successful, unique treatment option. Narrowing the gap between vision and current practice may appear complex, as clinical randomised controlled trials (RCTs) using such novel techniques are not yet available due to the limitations of each of the currently employed procedures. Yet, with significant advances in experimental work, some guidance can be proposed to reach a model method. Such a novel procedure should ideally fulfil the following conditions:

\section{Clinical requirements}

It should allow for a rapid increase in cartilage stability, restore joint function, alleviate pain and improve quality of life.

\section{Technical requirements}

It should be independent of the size of the lesion, applicable to chondral and osteochondral defects, and performed by arthroscopy.

\section{Requirements relative to current practice}

Clinical outcomes (pain and function) should be superior to the available options (be more controllable than microfracture, and more convenient and less expensive than ACI).

\section{The procedure}

Ideally, a translational procedure would combine in one single approach the best of each of the current techniques while minimising their limitations (Fig. 3). From a scientific point of view, the following actions might be envisaged to refine the current procedures:

\section{To improve microfracture}

A better control of chondrogenesis might be achieved by supplementing this procedure with a multifaceted scaffold (an intelligent biomaterial possibly supplemented with biological factors and cells to control and enhance repair) (Kon et al., 2012).

\section{To improve $A C I$}

Converting ACI to a one-step procedure by a technique such as that presented by Daniël Saris from the Utrecht team on the IMPACT one stage innovation during his presentation on the "Technovolution of cartilage repair". In this procedure which is under development and evaluation, autologous chondrons mixed with allogeneic mesenchymal 
stem cells in fibrin glue were administered to patients using a simple 75-min protocol in the operating room (Bekkers et al., 2013) (Fig. 4).

\section{The requirements}

Strict conditions will have to be applied when supplementing clinical procedures (Johnstone et al., 2013).

\section{Regarding the biomaterial}

The biomaterial should be an instructive, biodegradable scaffold that can direct the formation of extracellular matrix and that is immediately biomechanically functional to minimise post-operative unloading time. It should be capable of integrating with the surrounding chondral/ osteochondral tissue (multi-layered scaffold) to allow for the ingrowth, expansion, and differentiation of progenitor cells from the subchondral bone (access to marrow elements by preparation of the defect using microfracture, Pridie drilling, or abrasion) or from the joint environment (migration from the synovium or superficial cartilage layers). It should have an intrinsic ability to bind biological factors (or gene transfer vectors coding for biological factors) and ideally already be approved for use in patients by regulatory organisations. Technological details will have to be carefully considered and optimised to ensure ease of transfer from a laboratory setting to the clinic (ability to sterilise, easily formed/cut into different sizes, scalable for commercial production, storage for long periods of time, and be cost effective).

\section{Regarding the biologic factor}

The biologic factor (recombinant peptide or protein, or alternatively gene sequence with a gene transfer vector) should ideally also already be approved for use by regulatory organisations, so that the modified scaffold might be conveniently considered as a bioactive device rather than as a drug. At present, such approval only applies to a limited number of growth factors (e.g., BMP7 and PDGF).

\section{Regarding the cells}

Ideally, an acellular implant that is capable of attracting endogenous cells (e.g., as an adjunct to microfracture) would minimise the need for cell harvesting or ex vivo manipulation. Nonetheless, a variety of cell sources are potentially applicable, including terminally differentiated chondrocytes or alternatively progenitor cells. In this case, any cell source should also already be approved for use by regulatory organisations. The source and quality of cells remain to be defined (purified versus expanded versus mixed cell populations such as aspirates; autologous versus allogeneic cells; effects of age and disease on the maintenance and differentiation potential). Ideally, a unique source of cells applicable for all cases would be a major asset. In this respect, an allogeneic "universal donor" cell that has been screened for disease and properly typed to prevent immunogenic responses would be highly favoured. One possibility is an "immortalised non-tumorigenic cell line" possibly derived from induced pluripotent stem cells (iPSCs) (Takahashi et al., 2007), but it remains to be seen how such experimental work will translate into the clinic.
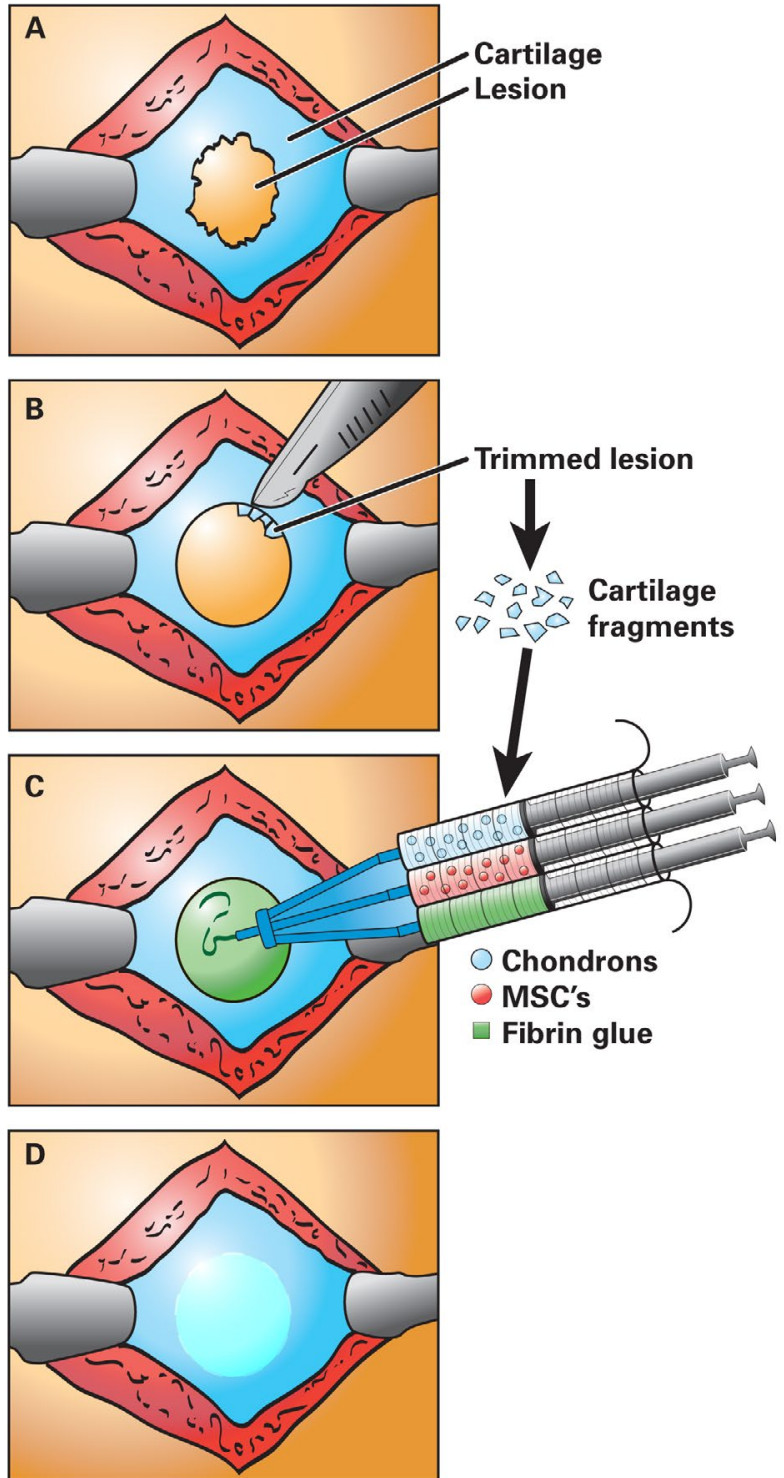

Fig. 4. The IMPACT one stage ACI. Cartilage lesions (A) are trimmed to leave smooth margins (B), and the resulting cartilage fragments are briefly proteolytically digested to release chondrons. The chondrons are injected together with allogeneic mesenchymal stem cells and fibrin glue into the trimmed cartilage lesion $(\mathbf{C})$, where they will give rise to a new articular cartilage (D).

\section{Conclusions and Notes of Caution}

The ideal procedure for articular cartilage repair is probably the one that will take advantage of the current approaches (ease of one-step microfracture; chondrogenic benefits of ACI) while improving their respective limitations (supplemented microfracture and simplified ACI). It will be critical to establish strict, careful rules to compare the current vision procedure with the classical surgical options to verify its potential benefits. It remains to be seen, though, whether the ultimate goal of such a therapy is to regenerate the original articular cartilage in patients (or merely to produce a long-lived, functional repair tissue), as the 
alleviation of pain and restoration of joint function and of quality of life are their essential expectations.

\section{Acknowledgements}

The authors would like to thank the AO Foundation for organising and funding the meeting, and Dr. Brigitte Vollmar and Dr. Tim Pohlemann for chairing the podium session upon which this workshop was based.

The abstracts from this meeting are available at: http://www.ecmjournal.org/ journal/supplements/ vol026supp08/AO13.htm

\section{Disclosure}

Dr. Guilak is a founder of Cytex Therapeutics, Inc. No other authors have anything to declare.

\section{References}

Bekkers JE, Inklaar M, Saris DB (2009) Treatment selection in articular cartilage lesions of the knee: a systematic review. Am J Sports Med 37 Suppl 1: 148S-155S.

Bekkers JE, Tsuchida AI, Van Rijen, Vonk LA, Dhert WJ, Creemers LB, Saris DB (2013) Single-stage cellbased cartilage regeneration using a combination of chondrons and mesenchymal stromal cells: comparison with microfracture. Am J Sports Med 41: 2158-2166.

Brittberg M (2008) Autologous chondrocyte implantation--technique and long-term follow-up. Injury 39 Suppl 1: S40-49.
Brittberg M (2010) Cell carriers as the next generation of cell therapy for cartilage repair: a review of the matrixinduced autologous chondrocyte implantation procedure. Am J Sports Med 38: 1259-1271.

Hjelle K, Solheim E, Strand T, Muri R, Brittberg M (2002) Articular cartilage defects in 1,000 knee arthroscopies. Arthroscopy 18: 730-734.

Johnstone B, Alini M, Cucchiarini M, Dodge GR, Eglin D, Guilak F, Madry H, Mata A, Mauck RL, Semino CE, Stoddart MJ (2013) Tissue engineering for articular cartilage repair - the state of the art. Eur Cell Mater 25: 248-267.

Kon E, Filardo G, Di Martino A, Marcacci M (2012) ACI and MACI. J Knee Surg 25: 17-22.

Madry H, Grun UW, Knutsen G (2011) Cartilage repair and joint preservation: medical and surgical treatment options. Dtsch Arztebl Int 108: 669-677.

Orth P, Cucchiarini M, Kohn D, Madry H (2013) Alterations of the subchondral bone in osteochondral repair - translational data and clinical evidence. Eur Cell Mater 25: 299-316.

Steadman JR, Rodkey WG, Rodrigo JJ (2001) Microfracture: surgical technique and rehabilitation to treat chondral defects. Clin Orthop Relat Res 391 Suppl: S362-369.

Takahashi K, Tanabe K, Ohnuki M, Narita M, Ichisaka T, Tomoda K, Yamanaka S (2007) Induction of pluripotent stem cells from adult human fibroblasts by defined factors. Cell 131: 861-872.

Editor's Note: All comments/questions by the reviewers were answered by making changes in the text. There is hence no Discussion with Reviewers section. 